\title{
Prediction of biomarker miRNAs signature in colorectal cancer metastasis to liver cancer
}

\author{
Z Salehi ${ }^{1}$, P Hadadi ${ }^{2}$, Omid Tavallaei ${ }^{1,2}$
}

\begin{abstract}
Introduction: microRNAs (miRNAs) are frequently dysregulated in colorectal cancer (CRC) primary tumors vs. metastasic to the liver. Objective: Our aim was to prediction of biomarker miRNAs signature of CRC Metastasis to liver cancer.

Material and Methods: mRNA and miRNA expression profiles of CRC primary tumors to metastases formed in the liver were downloaded from NCBI Gene Expression Omnibus (GEO) database. miRNAs targets were predicted using Targetscan algorithm. The Kyoto Encyclopedia of Genes and Genomes (KEGG) pathway and Gene Ontology (GO) enrichment analysis were performed using DAVID online tool, since mRNA expression profile of liver and colon cancer primary tumors vs. metastasis to the liver were used in background of these analysis.

Results: 43 and 58 down- and up-regulated miRNAs were obtained from GSE98406 (p-value $<0.05$ ). mRNA expression profile include of 1,048 mRNAs differentially expressed in metastatic and non-metastatic CRC to liver from GSE40367 ( $p$-value < 0.05 ). The some of the down-regulated miRNAs were significantly enriched in migration signaling and cancer stem cell signaling. Moreover, the some of the up-regulated miRNAs were significantly enriched in negative regulation of cell migration.

Conclusions: Some of the miRNAs are many number of target genes that some of these are oncogenes and tumor suppressor genes. It is concluded that differentially expressed miRNAs in metastatic vs. non-metastatic CRC to the liver take part in cell migration and cancer stem cell signaling pathways.
\end{abstract}

Keywords: miRNA expression data, mRNA expression data, signaling pathway, colorectal cancer, liver cancer

\section{INTRODUCTION}

MicroRNAs (miRNA) are short, 18-25 nucleotide-long, noncoding RNA molecules that regulate gene expression by suppressing mRNA translation whose dysregulation has been implicated in most of the cancers (1). miRNAs bind to the 3 ' noncoding region of the target mRNA and inhibit the expression of multiple target genes (2). Generally, oncogenic miRNAs (oncomiRs) are overexpressed while tumor-suppressive miRNAs are underexpressed in cancers (3). When these oncomiRs or tumor-suppressor miRNAs are stimulated or inhibited, respectively, cancer cell proliferation, metastasis, and/or survival may be significantly induced (3).

The incidence of colorectal cancer (CRC) is one of the highest rates of morbidity and mortality worldwide (4). Many studies are actively pursuing molecular biological analyses of the mechanisms involved in progression of colon cancer (4).

Biomarkers, such as coding and non-coding RNA are more remarkable and play significant roles in many biological processes (5). Many additional researches demonstrated that miRNAs are one of the most important types of cancer biomarkers and are therapeutic targets for CRC (5). Dysregulation of miRNAs were identified in CRC liver metastasis compared to primary CRC (6).

The biggest problem of cancer treatment is the spread of malignant cells from a primary tumor to distant sites or metastasis that is the most common cause of cancer-related mortality (7). Metastasis involves several continuous steps through which cancer cells disseminate and spread from a primary tumor to distant sites and forming secondary tumors in other tissues (7). Many of miRNAs play a critical role in pathological conditions, including tumorigenesis and metastasis

\footnotetext{
Department of pharmacognosy and pharmaceutical biotechnology, School of Pharmacy, Kermanshah University of Medical Sciences, Kermanshah, Iran.

2 Pharmaceutical Sciences Research Center, School of Pharmacy, Kermanshah University of Medical Sciences, Kermanshah, Iran.
}

Correspondence: Omid Tavallaei

Department of pharmacognosy and pharmaceutical biotechnology, School of Pharmacy, Kermanshah University of Medical Sciences, Kermanshah, Iran

E-mail: o.tavallaei@kums.ac.ir

Received: 11 Mar 2018, Accepted: 12 May 2018

(C) 2019 by the authors; licensee Modestum Ltd., UK. This article is an open access article distributed under the terms and conditions of the Creative Commons Attribution License (http://creativecommons.org/licenses/by/4.0/). 
Table 1: Description of microarray datasets

\begin{tabular}{llllc}
\hline Dataset & Type of samples & Group & Tissue & Number of samples \\
\hline Series GSE98406 & miRNA & Non-metastatic & Colon/liver & 14 \\
\hline Series GSE98406 & miRNA & Metastatic & Colon/liver & 28 \\
\hline Series GSE40367 & mRNA & Non-metastatic & tumor endothelium from colon adenocarcinoma tissue & 8 \\
\hline Series GSE40367 & mRNA & metastatic & tumor endothelium from liver metastasis & 7 \\
\hline
\end{tabular}

(8). Specific metastasis-regulating miRNAs, "metastamirs", govern molecular processes and pathways in malignant progression (8). However, research on the functions and mechanisms of microRNAs (miRNAs) in metastasis has only recently begun (8). So far, it has not been investigated CRC liver metastasis is being regulated by which important miRNAs. Therefore, our aim was to predict the miRNAs and molecular mechanisms of CRC liver metastasis.

\section{MATERIALS AND METHODS}

\section{Data Sources}

The mRNA expression profile of GSE40367 ( in metastatic vs. non-metastatic CRC to liver) was obtained from the National Center for Biotechnology Information (NCBI) Gene Expression Omnibus (GEO) database (http://www.ncbi.nlm.nih.gov/geo), that is based on the GPL570 [HG-U133_Plus_2] Affymetrix Human Genome U133 Plus 2.0 Array. On the other hand, comparing miRNA Signature of CRC primary tumors compare to metastases formed in the liver of GSE98406 was obtained from the GEO database, based on Affymetrix Multispecies miRNA-3 array. Description of microarray datasets was shown in Table 1. The row data were analyzed with GEO2R.

\section{miRNA Target Prediction}

Target genes of all miRNAs were predicted by Targetscan database with context score $<-0.2$ (http://www.targetscan.org). The predicted targets that became in GSE40367 were used for analysis.

\section{Pathway and Functional Enrichment Analyses}

Kyoto encyclopedia of genes and genomes (KEGG) pathway (9) and gene ontology (GO) (10) functional analyses were performed to identify significantly enriched pathways and the biological processes of miRNAs target genes, respectively, using the online tool of the database for annotation, visualization and integrated discovery (DAVID, http://david. abcc.ncifcrf.gov/) (11). mRNAs list in series GSE40367 ( $p$-value < 0.05) were uploaded into DAVID database as background tab. miRNAs target genes lists were uploaded into DAVID database. The entire human genome and mRNAs list in series GSE40367 were selected for the background control and we filtered out the specific gene expressed in metastatic vs. non-metastatic CRC to liver of series GSE40367 ( $p$-value < 0.05 ).

\section{RESULTS}

\section{Differentially Expressed mRNAs and miRNAs in Metastatic vs. Non-metastatic CRC to Liver}

In this study we collected the miRNA expression profile (Accession: GSE98406) and mRNA expression profiling (Accession: GSE40367) in GEO database. miRNA expression profile include of 43 and 58 down- and up-regulated miRNAs, respectively. mRNA expression profile include of 1,048 mRNAs differentially expressed in metastatic and non-metastatic CRC to liver ( $p$-value $<0.05$ ). The microarray datasets were descripted in Table 1 and miRNAs list and log FC for each miRNAs were shown in Table 2. 
Table 2: ID, names and fold change of up- and down-regulated miRNAs of metastatic vs. non-metastatic CRC to liver

\begin{tabular}{|c|c|c|c|c|c|}
\hline \multicolumn{3}{|c|}{ Up-regulated miRNAs } & \multicolumn{3}{|c|}{ Down-regulated miRNAs } \\
\hline miRNA_ID & P.Value & $\log \mathrm{FC}$ & miRNA_ID & P.Value & $\log \mathrm{FC}$ \\
\hline hsa-mir-200c & $4.87 \mathrm{e}-08$ & 3.4462 & hsa-mir-486 & $7.67 \mathrm{e}-11$ & -1.186 \\
\hline hsa-mir-182 & $1.38 \mathrm{e}-07$ & 1.738 & hsa-mir-451 & $4.54 \mathrm{e}-08$ & -1.09 \\
\hline hsa-mir-501 & $2.13 e-07$ & 0.2092 & hsa-mir-193a & $8.33 e-06$ & -0.8401 \\
\hline hsa-mir-1246 & $3.64 \mathrm{e}-06$ & 1.6368 & hsa-mir-1280 & $2.05 e-05$ & -0.9676 \\
\hline hsa-mir-3651 & $7.03 e-06$ & 0.1843 & hsa-mir-4454 & $6.07 e-05$ & -0.2395 \\
\hline hsa-mir-18a & $1.00 \mathrm{e}-05$ & 1.2815 & hsa-mir-4287 & $6.71 \mathrm{e}-05$ & -0.1317 \\
\hline hsa-mir-203 & $1.33 \mathrm{e}-05$ & 1.4432 & hsa-mir-630 & $1.00 \mathrm{e}-04$ & -0.0809 \\
\hline hsa-mir-18b & $1.44 \mathrm{e}-05$ & 0.5232 & hsa-mir-603 & $1.26 \mathrm{e}-04$ & -0.4742 \\
\hline hsa-mir-106b & $1.65 \mathrm{e}-05$ & 0.6524 & hsa-mir-1911 & $1.29 \mathrm{e}-04$ & -0.1571 \\
\hline hsa-mir-183 & $1.77 e-05$ & 0.4473 & hsa-mir-3199-1 & $1.47 e-04$ & -0.0569 \\
\hline hsa-mir-421 & $1.93 \mathrm{e}-05$ & 0.4535 & hsa-mir-3128 & $1.76 \mathrm{e}-04$ & -0.6054 \\
\hline hsa-mir-21 & $2.16 \mathrm{e}-05$ & 1.4669 & hsa-mir-4742 & $1.84 \mathrm{e}-04$ & -0.4358 \\
\hline hsa-mir-512-1 // hsa-mir-512-2 & $2.29 \mathrm{e}-05$ & 0.1037 & hsa-mir-4796 & $1.88 \mathrm{e}-04$ & -0.0729 \\
\hline hsa-mir-501 & $2.91 \mathrm{e}-05$ & 0.1166 & hsa-mir-720 & $2.96 \mathrm{e}-04$ & -0.9787 \\
\hline hsa-mir-552 & $3.50 \mathrm{e}-05$ & 0.525 & hsa-mir-122 & $3.27 e-04$ & -0.0989 \\
\hline hsa-mir-224 & $3.60 \mathrm{e}-05$ & 0.391 & hsa-mir-887 & $3.41 \mathrm{e}-04$ & -0.0802 \\
\hline hsa-mir-200a & $5.19 \mathrm{e}-05$ & 1.5269 & hsa-mir-4775 & $3.44 \mathrm{e}-04$ & -0.0863 \\
\hline hsa-mir-1179 & $6.14 \mathrm{e}-05$ & 0.0676 & hsa-mir-378 & $3.58 \mathrm{e}-04$ & -1.1897 \\
\hline hsa-mir-200b & $7.16 \mathrm{e}-05$ & 1.4889 & hsa-mir-488 & $3.73 e-04$ & -0.0933 \\
\hline hsa-mir-155 & $7.55 \mathrm{e}-05$ & 1.1209 & hsa-mir-3201 & $4.12 \mathrm{e}-04$ & -0.8948 \\
\hline hsa-mir-1291 & $1.09 \mathrm{e}-04$ & 0.0895 & hsa-mir-139 & $4.22 \mathrm{e}-04$ & -0.5518 \\
\hline hsa-mir-652 & $1.14 \mathrm{e}-04$ & 0.7115 & hsa-mir-193b & $4.79 e-04$ & -0.6085 \\
\hline hsa-mir-93 & $1.14 \mathrm{e}-04$ & 0.2957 & hsa-mir-548n & $6.38 e-04$ & -0.114 \\
\hline hsa-mir-141 & $1.21 \mathrm{e}-04$ & 1.4191 & hsa-mir-551b & $6.49 \mathrm{e}-04$ & -0.8187 \\
\hline hsa-mir-660 & $1.73 e-04$ & 0.535 & hsa-mir-4468 & $6.70 e-04$ & -0.0782 \\
\hline hsa-mir-1248 & $1.93 e-04$ & 0.3049 & hsa-mir-378f & $6.87 e-04$ & -0.0856 \\
\hline hsa-mir-210 & $2.33 e-04$ & 1.1209 & hsa-mir-4686 & $6.88 \mathrm{e}-04$ & -0.0988 \\
\hline hsa-mir-17 & $2.90 \mathrm{e}-04$ & 1.8819 & hsa-mir-4668 & $7.10 \mathrm{e}-04$ & -1.1861 \\
\hline hsa-mir-146a & $2.95 \mathrm{e}-04$ & 1.1806 & hsa-mir-320c-1 // hsa-mir-320c-2 & $8.57 e-04$ & -0.6253 \\
\hline hsa-mir-200c & $3.08 \mathrm{e}-04$ & 0.1468 & hsa-mir-378c & $8.67 e-04$ & -0.8827 \\
\hline hsa-mir-181b-1 // hsa-mir-181b-2 & $3.08 \mathrm{e}-04$ & 0.9151 & hsa-mir-4744 & $9.41 e-04$ & -0.0747 \\
\hline hsa-mir-3651 & $3.68 \mathrm{e}-04$ & 1.0025 & hsa-let-7c & $9.54 \mathrm{e}-04$ & -0.0733 \\
\hline hsa-mir-503 & $3.93 e-04$ & 0.4039 & hsa-mir-18b & $1.09 \mathrm{e}-03$ & -0.0609 \\
\hline hsa-mir-130b & $4.20 \mathrm{e}-04$ & 0.5855 & hsa-mir-3545 & $1.11 \mathrm{e}-03$ & -0.0781 \\
\hline hsa-mir-181d & $4.32 \mathrm{e}-04$ & 0.5109 & $\begin{array}{c}\text { hsa-mir-548i-1 // hsa-mir-548i-2 // hsa-mir- } \\
548 \mathrm{i}-3 / / \text { hsa-mir-548i-4 }\end{array}$ & $1.22 \mathrm{e}-03$ & -0.0622 \\
\hline hsa-mir-362 & $4.46 \mathrm{e}-04$ & 0.5768 & hsa-mir-3910-1 & $1.22 \mathrm{e}-03$ & -0.3205 \\
\hline hsa-mir-500a & $4.51 \mathrm{e}-04$ & 0.4431 & hsa-mir-4727 & $1.26 \mathrm{e}-03$ & -0.1704 \\
\hline hsa-mir-100 & $5.48 \mathrm{e}-04$ & 0.0625 & hsa-mir-204 & $1.26 \mathrm{e}-03$ & -0.069 \\
\hline hsa-mir-532 & $5.80 \mathrm{e}-04$ & 0.7376 & hsa-mir-548ai & $1.28 \mathrm{e}-03$ & -1.1277 \\
\hline hsa-mir-21 & $5.84 \mathrm{e}-04$ & 0.1499 & hsa-mir-378d-1 & $1.30 \mathrm{e}-03$ & -0.1319 \\
\hline hsa-mir-106b & $6.36 \mathrm{e}-04$ & 0.0988 & hsa-mir-320b-2 & $1.45 e-03$ & -0.0829 \\
\hline hsa-mir-93 & $6.56 \mathrm{e}-04$ & 1.0261 & hsa-mir-628 & $1.47 \mathrm{e}-03$ & -0.5139 \\
\hline hsa-mir-25 & $6.80 \mathrm{e}-04$ & 0.7632 & hsa-mir-518e & $1.47 \mathrm{e}-03$ & -0.0889 \\
\hline hsa-mir-221 & $7.01 \mathrm{e}-04$ & 1.3834 & & & \\
\hline hsa-mir-222 & $7.45 e-04$ & 1.3807 & & & \\
\hline hsa-mir-106a & $7.91 \mathrm{e}-04$ & 1.6868 & & & \\
\hline hsa-mir-20a & $8.78 \mathrm{e}-04$ & 1.6132 & & & \\
\hline hsa-mir-1301 & $9.96 \mathrm{e}-04$ & 0.2818 & & & \\
\hline hsa-mir-339 & $1.04 \mathrm{e}-03$ & 0.3614 & & & \\
\hline hsa-mir-4711 & $1.13 e-03$ & 0.0667 & & & \\
\hline hsa-mir-500a & $1.17 e-03$ & 0.3682 & & & \\
\hline $\begin{array}{c}\text { hsa-mir-1244-1 // hsa-mir-1244-2 // hsa- } \\
\text { mir-1244-3 }\end{array}$ & $1.20 \mathrm{e}-03$ & 0.1371 & & & \\
\hline hsa-mir-1247 & $1.29 \mathrm{e}-03$ & 0.0922 & & & \\
\hline hsa-mir-17 & $1.34 \mathrm{e}-03$ & 0.3437 & & & \\
\hline hsa-mir-19a & $1.39 \mathrm{e}-03$ & 0.3013 & & & \\
\hline hsa-mir-20b & $1.39 \mathrm{e}-03$ & 1.0308 & & & \\
\hline hsa-mir-378d-2 & $1.41 \mathrm{e}-03$ & 0.0574 & & & \\
\hline hsa-mir-1290 & $1.43 e-03$ & 0.4762 & & & \\
\hline
\end{tabular}




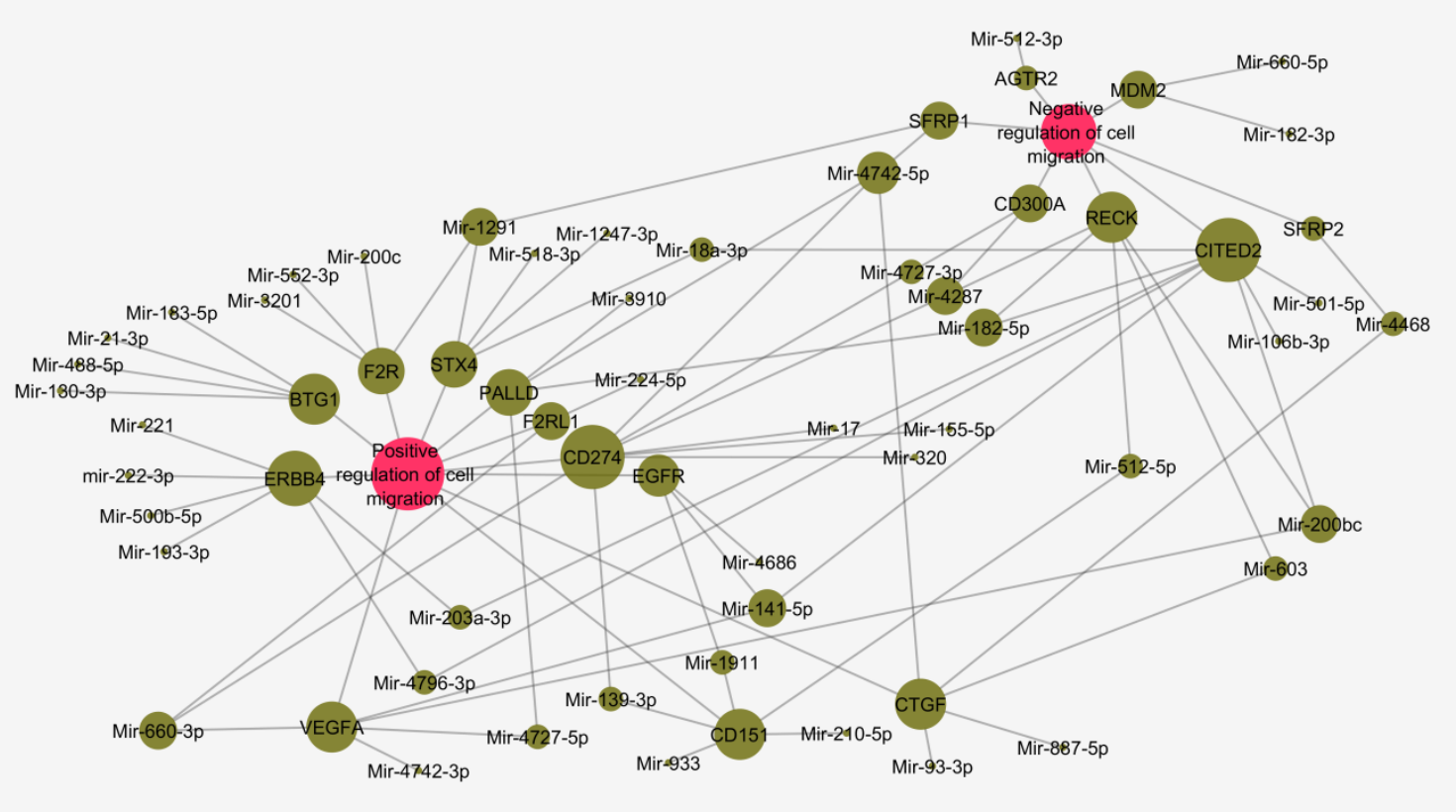

Figure 1: Overall survival time by stage

\section{Identification of miRNAs Target Genes}

Target genes of miRNAs were identified by Targetscan database (with total context score $<-0.2$ ). The number of miRNAs target genes were 30,630 and 24,435 for up- and down-regulated miRNAs in metastatic vs. non-metastatic CRC to liver, respectively.

\section{Biological Process Analysis}

In order to investigate the roles of miRNAs target genes, gene ontology (GO) biological processes (BP) module of the DAVID database were used. The GO BP results indicate that the some of the target genes of some down-regulated miRNAs are enriched to leukocyte migration, fibroblast migration, neuron migration, germ cell migration and regulation of endothelial cell migration. For example, ATP1B3, SLC16A1, KITLG and CD44, the target genes of mir-603, mir-4727$5 p$, mir-3201 and mir-204-3p, respectively, take part in leukocyte migration. The some of the results were shown in Figure 1.

In contrast, the some of the target genes of many up-regulated miRNAs take part in negative regulation of cell migration. For instance, mir-200bc, mir-182-5p, mir-501-5p, mir-203a-3p, mir-501-5p and mir-141-5p involved in negative regulation of cell migration by targeting CITED2.

Many of target genes were targeted by both up- and down-regulated miRNAs and involved in both negative and positive cell migration. The complete GO BP results, miRNAs and their target genes were shown in Table 3. 
Table 3: MiRNAs, their target genes and GO BP results of up-and down-regulated miRNAs of metastatic vs. non-metastatic $C R C$ to liver

\begin{tabular}{|c|c|c|c|}
\hline miRNA & Regulation & Target Genes & Biological Process \\
\hline Mir-182-5p & Up & \multirow{5}{*}{ ATPase $\mathrm{Na}+/ \mathrm{K}+$ transporting subunit beta $3(\mathrm{ATP} 1 \mathrm{~B} 3$ ) } & \multirow{40}{*}{ leukocyte migration } \\
\hline Mir-603 & down & & \\
\hline Mir-224-5p & up & & \\
\hline Mir-155-3p & up & & \\
\hline Mir-1301-3p & up & & \\
\hline Mir-200bc & Up & \multirow{4}{*}{ fibronectin 1(FN1) } & \\
\hline Mir-183-5p & up & & \\
\hline Mir-155-3p & up & & \\
\hline Mir-146a-3p & up & & \\
\hline Mir-887-5p & Down & \multirow{4}{*}{ solute carrier family 16 member $1(\mathrm{SLC} 16 \mathrm{~A} 1)$} & \\
\hline Mir-21-3p & up & & \\
\hline Mir-4727-5p & down & & \\
\hline Mir-1291 & up & & \\
\hline Mir-1911 & Down & \multirow{6}{*}{ KIT ligand(KITLG) } & \\
\hline Mir-3201 & Down & & \\
\hline Mir-320 & Down & & \\
\hline Mir-4727-3p & Down & & \\
\hline Mir-200c & Up & & \\
\hline Mir-200ab & Up & & \\
\hline Mir-1911 & Down & \multirow{7}{*}{ CD44 molecule (Indian blood group)(CD44) } & \\
\hline Mir-488-5p & Down & & \\
\hline Mir-18-3p & Down & & \\
\hline Mir-204-3p & Down & & \\
\hline Mir-518d & Down & & \\
\hline Mir-18b-3p & Up & & \\
\hline Mir-652-5p & Up & & \\
\hline Mir-3201 & Down & \multirow{9}{*}{ CD48 molecule(CD48) } & \\
\hline Mir-18-3p & Down & & \\
\hline Mir-4727-5p & Down & & \\
\hline Mir-548ai & Down & & \\
\hline Mir-3651 & Up & & \\
\hline Mir-18a-3p & Up & & \\
\hline Mir-18b-3p & Up & & \\
\hline Mir-21-3p & Up & & \\
\hline Mir-3651 & Up & & \\
\hline Mir-193-5p & Down & \multirow{5}{*}{ CD34 molecule(CD34) } & \\
\hline Mir-3651 & Up & & \\
\hline Mir-203a-5p & Up & & \\
\hline Mir-552-3p & Up & & \\
\hline Mir-3651 & up & & \\
\hline Mir-4727-5p & Down & \multirow{2}{*}{ promyelocytic leukemia(PML) } & \multirow{2}{*}{ fibroblast migration } \\
\hline Mir-1291 & Up & & \\
\hline Mir-193a & Down & \multirow{6}{*}{ BTG anti-proliferation factor 1(BTG1) } & \\
\hline Mir-488-5p & Down & & \\
\hline Mir-4646-5p/mir-204-3p & Down & & \\
\hline Mir-183-5p & Up & & \\
\hline Mir-21-3p/mir-3591-3p & Up & & \\
\hline Mir-130-3p & Up & & \\
\hline Mir-4742-3p & Down & \multirow{5}{*}{ vascular endothelial growth factor A(VEGFA) } & \\
\hline Mir-4727-5p & Down & & \\
\hline Mir-200bc & Up & & \\
\hline Mir-141-5p & Up & & \\
\hline Mir-660-3p & Up & & \\
\hline Mir-4742-5p & Down & \multirow{4}{*}{ palladin, cytoskeletal associated protein(PALLD) } & coll minrotion \\
\hline Mir-3910 & Down & & cell migration \\
\hline Mir-4727-5p & Down & & \\
\hline Mir-182-5p & up & & \\
\hline Mir-518-3p & Down & \multirow{4}{*}{ syntaxin 4(STX4) } & \\
\hline Mir-18a-3p & Up & & \\
\hline Mir-1291/mir-6775 & Up & & \\
\hline Mir-1247-3p & up & & \\
\hline Mir-193-3p & Down & & \\
\hline Mir-4796-3p & Down & & \\
\hline Mir-203a-3p & Up & erb-b2 receptor tyrosine kinase $4($ ERBB4) & \\
\hline Mir-500b-5p & Up & & \\
\hline Mir-221/mir-222-3p & Up & & \\
\hline
\end{tabular}


Table 3 (continued): MiRNAs, their target genes and GO BP results of up-and down-regulated miRNAs of metastatic vs. non-metastatic CRC to liver

\begin{tabular}{|c|c|c|c|}
\hline miRNA & Regulation & Target Genes & Biological Process \\
\hline Mir-1911 & Down & \multirow{3}{*}{ epidermal growth factor receptor(EGFR) } & \multirow{27}{*}{ cell migration } \\
\hline Mir-4686 & Down & & \\
\hline Mir-141-5p & Up & & \\
\hline Mir-603 & Down & \multirow{5}{*}{ connective tissue growth factor(CTGF) } & \\
\hline Mir-4742-5p & Down & & \\
\hline Mir-887-5p & Down & & \\
\hline Mir-4468 & Down & & \\
\hline Mir-93-3p & Up & & \\
\hline Mir-224-5p & Up & \multirow{2}{*}{ F2R like trypsin receptor 1(F2RL1) } & \\
\hline Mir-660-3p & Up & & \\
\hline Mir-200c & Up & \multirow{4}{*}{ coagulation factor II thrombin receptor(F2R) } & \\
\hline Mir-552-3p & Up & & \\
\hline Mir-1291 & Up & & \\
\hline Mir-3201 & Down & & \\
\hline Mir-4287 & Down & \multirow{8}{*}{ CD274 molecule(CD274) } & \\
\hline Mir-4742-5p & Down & & \\
\hline Mir-139-3p & Down & & \\
\hline Mir-320 & Down & & \\
\hline Mir-4727-3p & Down & & \\
\hline Mir-17 & Up & & \\
\hline Mir-155-5p & Up & & \\
\hline Mir-660-3p & Up & & \\
\hline Mir-1911 & Down & \multirow{5}{*}{ CD151 molecule (Raph blood group)(CD151) } & \\
\hline Mir-139-3p & Down & & \\
\hline Mir-933 & Down & & \\
\hline Mir-512-5p & Up & & \\
\hline Mir-210-5p & Up & & \\
\hline Mir-4287 & Down & \multirow{2}{*}{ CD300a molecule(CD300A) } & \multirow{2}{*}{$\begin{array}{l}\text { negative regulation of eosinophil } \\
\text { migration, }\end{array}$} \\
\hline Mir-4727-3p & Down & & \\
\hline Mir-4796-3p & Down & \multirow{9}{*}{$\begin{array}{l}\mathrm{Cbp} / \mathrm{p} 300 \text { interacting transactivator with Glu/Asp rich carboxy-terminal domain } \\
\qquad 2(\mathrm{CITED} 2)\end{array}$} & \multirow{20}{*}{$\begin{array}{l}\text { negative regulation of cell } \\
\text { migration }\end{array}$} \\
\hline Mir-200bc & Up & & \\
\hline Mir-182-5p & Up & & \\
\hline Mir-501-5p & Up & & \\
\hline Mir-18a-3p & Up & & \\
\hline Mir-203a-3p & Up & & \\
\hline Mir-106b-3p & Up & & \\
\hline Mir-501-5p & Up & & \\
\hline Mir-141-5p & Up & & \\
\hline Mir-4468 & Down & secreted frizzled related protein 2(SFRP2) & \\
\hline Mir-4287 & Down & \multirow{5}{*}{ reversion inducing cysteine rich protein with kazal motifs(RECK) } & \\
\hline Mir-603 & Down & & \\
\hline Mir-200bc & Up & & \\
\hline Mir-182-5p & Up & & \\
\hline Mir-512-5p & Up & & \\
\hline Mir-4742-5p & Down & \multirow{2}{*}{ secreted frizzled related protein 1(SFRP1) } & \\
\hline Mir-1291 & Up & & \\
\hline Mir-512-3p & Up & angiotensin II receptor type 2(AGTR2) & \\
\hline Mir-182-3p & Up & \multirow{2}{*}{ MDM2 proto-oncogene(MDM2) } & \\
\hline Mir-660-5p & Up & & \\
\hline Mir-1291 & up & doublecortin like kinase 1(DCLK1) & \\
\hline Mir-1911-5p & Down & & \\
\hline Mir-122-3p & Down & & \\
\hline Mir-200c & Up & mvocyte enhancer factor 2C(MEF2C) & \\
\hline Mir-182-5p & Up & 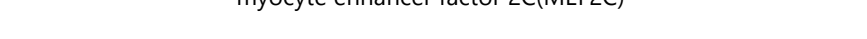 & \\
\hline Mir-501-5p & Up & & \\
\hline mir-501-3p & Up & & \\
\hline Mir-1911 & down & fibroblast growth factor receptor 1(FGFR1) & neuron miaration \\
\hline Mir-320 & down & delta/notch like EGF repeat containing(DNER) & menton mingration \\
\hline Mir-603 & Down & & \\
\hline Mir-183-5p & Up & & \\
\hline $\operatorname{mir}-660-3 p$ & Up & & \\
\hline Mir-1911 & Down & cyclin dependent kinase 5 regulatory subunit $1(C D K 5 R 1)$ & \\
\hline Mir-3201 & Down & & \\
\hline Mir-320 & Down & & \\
\hline Mir-4727-3p & Down & & \\
\hline
\end{tabular}


Table 3 (continued): MiRNAs, their target genes and GO BP results of up-and down-regulated miRNAs of metastatic vs. non-metastatic CRC to liver

\begin{tabular}{|c|c|c|c|}
\hline miRNA & Regulation & Target Genes & Biological Process \\
\hline Mir-200c & Up & \multirow{2}{*}{ cyclin dependent kinase 5 regulatory subunit 1(CDK5R1) } & \multirow{2}{*}{ neuron migration } \\
\hline Mir-200ab & Up & & \\
\hline Mir-933 & Down & KIT proto-oncogene receptor tyrosine kinase(KIT) & germ cell migration \\
\hline Mir-130b-5p & Up & SET domain containing 2(SETD2) & $\begin{array}{c}\text { cell migration involved in } \\
\text { vasculogenesis }\end{array}$ \\
\hline Mir-3910 & Down & \multirow{2}{*}{ SP100 nuclear antigen(SP100) } & negative regulation of endothelial \\
\hline Mir-200bc & Up & & cell migration \\
\hline Mir-603 & Down & \multirow{3}{*}{ epithelial membrane protein 2(EMP2) } & \multirow{16}{*}{$\begin{array}{l}\text { regulation of endothelial cell } \\
\text { migration }\end{array}$} \\
\hline Mir-183-5p & Up & & \\
\hline mir-660-3p & Up & & \\
\hline Mir-1911 & Down & \multirow{6}{*}{ phosphatidylinositol-4,5-bisphosphate 3-kinase catalytic subunit alpha(PIK3CA) } & \\
\hline Mir-3201 & Down & & \\
\hline Mir-320 & Down & & \\
\hline Mir-4727-3p & Down & & \\
\hline Mir-200c & Up & & \\
\hline Mir-200ab & Up & & \\
\hline Mir-3201 & Down & \multirow{2}{*}{ fibroblast growth factor 1 (FGF1) } & \\
\hline Mir-1301-3p & up & & \\
\hline Mir-4668 & Down & \multirow{2}{*}{ forkhead box C2(FOXC2) } & \\
\hline Mir-548ai & Down & & \\
\hline Mir-887-5p & Down & \multirow{3}{*}{ forkhead box P1(FOXP1) } & \\
\hline Mir-183-5p & Up & & \\
\hline Mir-93-3p & Up & & \\
\hline
\end{tabular}

Table 4: MiRNAs, their target genes and KEGG pathway results of up- and down-regulated miRNAs of metastatic vs. nonmetastatic CRC to liver

\begin{tabular}{|c|c|c|c|}
\hline miRNA & regulation & Target gene & Signaling pathway \\
\hline Mir-4742-3p & Down & \multirow{11}{*}{ G protein subunit gamma $12(G N G 12)$} & \\
\hline Mir-887-5p & Down & & \\
\hline Mir-4468 & Down & & \\
\hline Mir-548 & Down & & \\
\hline Mir-4668-5p & Down & & \\
\hline Mir-4744 & Down & & PI3K-Akt signaling pathway \\
\hline Mir-183-5p & Up & & \\
\hline Mir-512-5p & Up & & \\
\hline Mir-552-3p & Up & & \\
\hline Mir-503-3p & Up & & \\
\hline Mir-1244 & Up & & \\
\hline Mir-1911 & Down & \multirow{6}{*}{ KIT ligand(KITLG) } & \\
\hline Mir-3201 & Down & & \\
\hline Mir-320 & Down & & \\
\hline Mir-4727-3p & Down & & \\
\hline Mir-200c & Up & & \\
\hline Mir-200ab & Up & & \\
\hline Mir-933 & Down & KIT proto-oncogene receptor tyrosine kinase(KIT) & \\
\hline Mir-182-3p & Up & \multirow{2}{*}{ MDM2 proto-oncogene(MDM2) } & \\
\hline Mir-660-5p & Up & & \\
\hline Mir-5440-3p & Down & \multirow{3}{*}{ Raf-1 proto-oncogene, serine/threonine kinase(RAF1) } & \\
\hline Mir-4468 & Down & & \\
\hline Mir-146a-3p & Up & & \\
\hline
\end{tabular}

\section{Signaling Pathways Analysis}

We performed the KEGG pathways enrichment analysis for miRNAs target genes that up- or down-expressed in metastatic vs. non-metastatic CRC to liver. KEGG pathway indicated that some of the up-regulated miRNAs such as mir182-3p, mir-660-3p, mir, mir-4711-3p and mir-25 take part in tight junction, gap junction and adherens junction. Some of the up-regulated miRNAs such as mir-1291 and mir 1244 involved in cancer stem cells signaling as MAPK signaling pathway and PI3K-Akt signaling pathway.

But some of the down-regulated miRNAs in metastatic vs. non-metastatic CRC to liver such as mir-4742-3p, mir-8875p, mir-4468, mir-933, mir-1911 and mir-4668 involved in MAPK, Notch, Wnt and PI3K-Akt signaling pathway. Moreover, some of the down-regulated miRNAs act in cell junctions. The complete results of KEGG pathway for each up- and downregulated miRNAs target genes were shown in Table 4. 
Table 4 (continued): MiRNAs, their target genes and KEGG pathway results of up-and down-regulated miRNAs of metastatic vs. non-metastatic CRC to liver

\begin{tabular}{|c|c|c|c|}
\hline miRNA & regulation & Target gene & Signaling pathway \\
\hline Mir-139-3p & Down & SGK2, serine/threonine kinase 2(SGK2) & \\
\hline Mir-200c & Up & & \\
\hline Mir-552-3p & Up & \multirow{3}{*}{ coagulation factor II thrombin receptor(F2R) } & \\
\hline Mir-1291 & Up & & \\
\hline Mir-3201 & Down & & \\
\hline Mir-603 & Down & \multirow{2}{*}{ collagen type IV alpha 3 chain(COL4A3) } & \\
\hline Mir-548 & Down & & \\
\hline Mir-18a-3p & Up & collagen type XXVII alpha 1 chain(COL27A1) & \\
\hline Mir-106b-3p & Up & \multirow{3}{*}{ cyclin E1(CCNE1) } & \\
\hline Mir-1179 & Up & & \\
\hline Mir-3910 & Down & & \\
\hline Mir-4468 & Down & \multirow{3}{*}{ ephrin $\mathrm{A} 3(\mathrm{EFNA} 3$ ) } & \\
\hline Mir-210-3p & Up & & \\
\hline Mir-210-5p & Up & & \\
\hline Mir-141-5p & Up & eukaryotic translation initiation factor 4E family member 2(EIF4E2) & \\
\hline Mir-3201 & Down & \multirow{2}{*}{ fibroblast growth factor 1 (FGF1) } & \\
\hline Mir-1301-3p & up & & \\
\hline \multicolumn{4}{|c|}{ fibroblast growth factor 4(FGF4) } \\
\hline Mir-1911 & down & fibroblast growth factor receptor 1(FGFR1) & \\
\hline Mir-200bc & Up & \multirow{4}{*}{ fibronectin 1(FN1) } & \\
\hline Mir-183-5p & up & & \\
\hline Mir-155-3p & up & & \\
\hline Mir-146a-3p & up & & \\
\hline Mir-887-5p & Down & \multirow{5}{*}{ interleukin 2 receptor subunit alpha(IL2RA) } & \\
\hline Mir-4727-5p & Down & & \\
\hline Mir-512-3p & Up & & \\
\hline Mir-329-3p & Up & & \\
\hline Mir-500a-3p & Up & & \\
\hline Mir-4742-3p & Down & \multirow{7}{*}{ lysophosphatidic acid receptor 1(LPAR1) } & \\
\hline Mir-3910 & Down & & \\
\hline Mir-200bc & Up & & \\
\hline Mir-501-5p & Up & & \\
\hline Mir-3651 & Up & & \\
\hline Mir-660-3p & Up & & \\
\hline Mir-503-3p & Up & & \\
\hline Mir-1911 & Down & \multirow{6}{*}{$\begin{array}{l}\text { phosphatidylinositol-4,5-bisphosphate 3-kinase catalytic subunit } \\
\text { alpha(PIK3CA) }\end{array}$} & \\
\hline Mir-3201 & Down & & \\
\hline Mir-320 & Down & & \\
\hline Mir-4727-3p & Down & & \\
\hline Mir-200c & Up & & \\
\hline Mir-200ab & Up & & \\
\hline Mir-4287 & Down & \multirow{3}{*}{ toll like receptor $4(T L R 4)$} & \\
\hline Mir-630 & Down & & \\
\hline Mir-3201 & Down & & \\
\hline Mir-4742-3p & Down & \multirow{5}{*}{ vascular endothelial growth factor A(VEGFA) } & \\
\hline Mir-4727-5p & Down & & \\
\hline Mir-200bc & Up & & \\
\hline Mir-141-5p & Up & & \\
\hline Mir-660-3p & Up & & \\
\hline Mir-1291 & Up & ECSIT signalling integrator(ECSIT) & MAPK signaling pathway \\
\hline Mir-139-3p & Down & \multirow{2}{*}{ ELK1, ETS transcription factor(ELK1) } & \\
\hline Mir-660-5p & Up & & \\
\hline Mir-4742-3p & Down & & \\
\hline Mir-887-5p & Down & & \\
\hline Mir-4468 & Down & & \\
\hline Mir-548 & Down & & \\
\hline Mir-4668-5p & Down & & \\
\hline Mir-4744 & Down & G protein subunit gamma $12(G N G 12)$ & \\
\hline Mir-183-5p & Up & & \\
\hline Mir-512-5p & Up & & \\
\hline Mir-552-3p & Up & & \\
\hline Mir-503-3p & Up & & \\
\hline Mir-1244 & Up & & \\
\hline Mir-5440-3p & Down & & \\
\hline Mir-4468 & Down & Raf-1 proto-oncogene, serine/threonine kinase(RAF1) & \\
\hline Mir-146a-3p & Up & & \\
\hline Mir-3910 & Down & arrestin beta 1(ARRB1) & \\
\hline
\end{tabular}


Table 4 (continued): MiRNAs, their target genes and KEGG pathway results of up-and down-regulated miRNAs of metastatic vs. non-metastatic CRC to liver

\begin{tabular}{|c|c|c|c|}
\hline miRNA & regulation & Target gene & Signaling pathway \\
\hline Mir-17 & Up & calcium voltage-gated channel auxiliary subunit gamma 1(CACNG1) & \\
\hline Mir-3128 & Down & & \\
\hline Mir-1291 & Up & calcium voltage-gated channel auxiliary subunit gamma $4(\mathrm{CACNG} 4)$ & \\
\hline Mir-1247-3p & Up & & \\
\hline Mir-122-5p & Down & \multirow{2}{*}{ dual specificity phosphatase 2(DUSP2) } & \\
\hline Mir-4727-5p & Down & & \\
\hline Mir-1911 & Down & \multirow{3}{*}{ epidermal growth factor receptor(EGFR) } & \\
\hline Mir-4686 & Down & & \\
\hline Mir-141-5p & Up & & \\
\hline \multicolumn{4}{|c|}{ fibroblast growth factor 1 (FGF1) } \\
\hline \multicolumn{4}{|c|}{ fibroblast growth factor 4(FGF4) } \\
\hline Mir-1911 & down & fibroblast growth factor receptor 1(FGFR1) & \\
\hline Mir-1911-5p & Down & & \\
\hline Mir-4686 & Down & & \\
\hline Mir-4668-5p & Down & & \\
\hline Mir-548a & Down & mitogen-activated protein kinase kinase kinase 2(MAP3K2) & \\
\hline Mir-203a-5p & Up & & \\
\hline Mir-183-5p & Up & & \\
\hline Mir-1244 & Up & & \\
\hline Mir-887-5p & Down & mitogen-activated protein kinase kinase kinase kinase 1(MAP4K1) & \\
\hline & Down & \multirow{6}{*}{ myocyte enhancer factor $2 \mathrm{C}(\mathrm{MEF} 2 \mathrm{C})$} & \\
\hline Mir-1911-5p & Down & & \\
\hline Mir- $628-5 n$ & Down & & \\
\hline MIIr-628-5p & Up & & \\
\hline $\begin{array}{l}\text { Mir-200c } \\
\text { Mir-182-5n }\end{array}$ & Up & & \\
\hline $\begin{array}{l}\text { Mir-182-5p } \\
\text { Mir-501-5p }\end{array}$ & Up & & \\
\hline Mir-93-3p & Up & nuclear factor of activated T-cells 3(NFATC3) & \\
\hline Mir-203a-5p & Up & \multirow{2}{*}{ ribosomal protein S6 kinase A2(RPS6KA2) } & \\
\hline Mir-106b-3p & Up & & \\
\hline Mir-193-3p & Down & \multirow{5}{*}{ stathmin 1(STMN1) } & \\
\hline Mir-3201 & Down & & \\
\hline Mir-4668-5p & Down & & \\
\hline Mir-200ab & Up & & \\
\hline Mir-155-3p & Up & & \\
\hline Mir-1911 & Down & \multirow{3}{*}{$\begin{array}{l}\text { RFNG O-fucosylpeptide 3-beta-N- } \\
\text { acetylglucosaminyltransferase(RFNG) }\end{array}$} & \\
\hline Mir-933 & Down & & Notch signaling pathway \\
\hline Mir-93-3p & Up & & \\
\hline Mir-1911-5p & Down & \multirow{12}{*}{ Wnt family member $2 \mathrm{~B}(\mathrm{WNT} 2 \mathrm{~B})$} & \multirow{12}{*}{ Wnt signaling pathway } \\
\hline Mir-4742-5p & Down & & \\
\hline Mir-887-5p & Down & & \\
\hline Mir-488-5p & Down & & \\
\hline Mir-4686 & Down & & \\
\hline Mir-4668-5p & Down & & \\
\hline Mir-501-5p & Up & & \\
\hline Mir-18a & Up & & \\
\hline Mir-18b & Up & & \\
\hline Mir-501-5p & Up & & \\
\hline Mir-1179 & Up & & \\
\hline Mir-652-5p & Up & & \\
\hline Mir-3910 & Down & \multirow{2}{*}{ axin 2(AXIN2) } & \\
\hline Mir-1246 & Up & & \\
\hline \multicolumn{4}{|c|}{ frizzled class receptor 2(FZD2) } \\
\hline Mir-93-3p & Up & nuclear factor of activated T-cells 3(NFATC3) & \\
\hline Mir-182-3p & Up & LLGL1, scribble cell polarity complex component(LLGL1) & Tight junction \\
\hline Mir-3201 & Down & \multirow{3}{*}{ myosin heavy chain 11(MYH11) } & \\
\hline Mir-660-3p & Up & & \\
\hline Mir-4711-3p & Up & & \\
\hline \multicolumn{4}{|c|}{ myosin heavy chain $3(\mathrm{MYH} 3)$} \\
\hline Mir-887-5p & down & myosin light chain, phosphorylatable, fast skeletal muscle(MYLPF) & \\
\hline Mir-887-5p & Down & & \\
\hline Mir-4668-5p & Down & & \\
\hline Mir-3910 & Down & & \\
\hline Mir-203a-5p & Up & claudin 11(CLDN11) & \\
\hline Mir-421 & Up & & \\
\hline Mir-501/mir-502 & Up & & \\
\hline Mir-25-5p & Up & & \\
\hline
\end{tabular}


Table 4 (continued): MiRNAs, their target genes and KEGG pathway results of up-and down-regulated miRNAs of metastatic vs. non-metastatic CRC to liver

\begin{tabular}{|c|c|c|c|}
\hline miRNA & regulation & Target gene & Signaling pathway \\
\hline Mir-4744 & Down & \multirow{2}{*}{ claudin 14(CLDN14) } & \\
\hline Mir-660-3p & Up & & \\
\hline Mir-628-3p & Down & \multirow[t]{2}{*}{ catenin alpha 1 (CTNNA1) } & \\
\hline Mir-1179 & Up & & \\
\hline Mir-122-3p & Down & \multirow{6}{*}{ RAB3B, member RAS oncogene family(RAB3B) } & \\
\hline Mir-501-5p & Up & & \\
\hline Mir-512-3p & Up & & \\
\hline Mir-660-3p & Up & & \\
\hline Mir-210-5p & Up & & \\
\hline Mir-146a-3p & Up & & \\
\hline Mir-5440-3p & Down & \multirow{3}{*}{ Raf-1 proto-oncogene, serine/threonine kinase(RAF1) } & \\
\hline Mir-4468 & Down & & Gap junction \\
\hline Mir-146a-3p & Up & & \\
\hline Mir-1911 & Down & \multirow{3}{*}{ epidermal growth factor receptor(EGFR) } & \\
\hline Mir-4686 & Down & & \\
\hline Mir-141-5p & Up & & \\
\hline Mir-4468 & Down & \multirow{2}{*}{ adenylate cyclase 2(ADCY2) } & \\
\hline Mir-501 & Up & & \\
\hline Mir-628-3p & Down & \multirow{2}{*}{ catenin alpha 1(CTNNA1) } & Adherens junction \\
\hline Mir-1179 & Up & & Adherens junction \\
\hline Mir-1911 & Down & \multirow{3}{*}{ epidermal growth factor receptor(EGFR) } & \\
\hline Mir-4686 & Down & & \\
\hline Mir-141-5p & Up & & \\
\hline Mir-1911 & down & fibroblast growth factor receptor 1(FGFR1) & \\
\hline
\end{tabular}

\section{DISCUSSION}

According to the gene expression profile analysis between metastatic vs. non-metastatic CRC to liver groups, 58 upregulated and 55 down-regulated miRNAs in metastatic vs. non-metastatic CRC to liver were obtained (P.Value $<0.05$ ). On the other hand, 1,048 mRNAs differentially expressed in metastatic vs. non-metastatic CRC to liver were selected (P.Value < 0.05). After target prediction by targetscan algorithm, the miRNAs target genes that become in series GSE40367 were selected and GO BO and signaling pathways were analyzed by DAVID database. Since multiple miRNAs target the same gene, that determines the expression of miRNA target genes, one of the miRNAs targeted many numbers of mRNAs. Recent study indicated that hsa-mir-21 is an oncomir in CRC which in turn regulates the development of CRC (12). These results indicated that after up-regulation of miR-21 expression, the levels of AKT and PI3K protein expression significantly increased (12). Our results were shown that has-mir-21 upregulated in CRC and take part in cell migration. Evidences showed that has-mir-17 contributed to the proliferation and invasion of colorectal cancer and played a critical role in the proliferation and invasion of colorectal cancer (13). We showed that has-mir-17 were up-regulated in CRC and involved in cell migration (14). Moreover, levels of miR-21, and miR-155 increased significantly in human colon tumor samples, compared with normal tissues that have been associated with tumor growth (14). In these studies, results indicated that has-mir-155 was up-regulated in CRC and take part in PI3K-Akt signaling pathway and cell migration. Jun Qin et al. suggested that has-mir-221 promotes CRC cell invasion and metastasis (15). In our study, has-mir-221 as an up-regulated miRNAs was enriched in some biological processes in cell migration. Our results were shown that some of the miRNAs are many number of target genes that some of these are oncogenes and some are tumor suppressor genes. Also, our results indicated that differentially expressed miRNAs in metastatic vs. nonmetastatic CRC to the liver take part in cell migration and cancer stem cell signaling pathways. The target genes of some of the miRNAs promote negative regulation of cell migration. The results of bioinformatics analysis indicated that many of up- and down-regulated miRNAs involve in cancer stem cell processing in metastatic vs. non-metastatic CRC to liver that were shown in Table 3 and 4.

In conclusion, our study showed that differentially expressed miRNAs in metastatic vs. non-metastatic CRC to the liver take part in cell migration and cancer stem cell signaling pathways. 


\section{REFERENCES}

1. Calin GA, Croce CM. MicroRNA signatures in human cancers. Nature reviews cancer, 2006;6(11):857-866. https://doi.org/10.1038/nrc1997

2. Kim VN. MicroRNA biogenesis: coordinated cropping and dicing. Nature reviews Molecular cell biology, 2005;6(5):376-385. https://doi.org/10.1038/nrm1644

3. Banno K., et al. MicroRNA in cervical cancer: OncomiRs and tumor suppressor miRs in diagnosis and treatment. The Scientific World Journal. 2014. https://doi.org/10.1155/2014/178075

4. Ricci-Vitiani L., et al. Identification and expansion of human colon-cancer-initiating cells. Nature, 2007;445(7123):111-115. https://doi.org/10.1038/nature05384

5. Chen X., et al. Characterization of microRNAs in serum: a novel class of biomarkers for diagnosis of cancer and other diseases. Cell research. 2008;18(10):997-1006. https://doi.org/10.1038/cr.2008.282

6. Luo X., et al. MicroRNA signatures: novel biomarker for colorectal cancer? Cancer Epidemiology and Prevention Biomarkers, 2011. 20(7): p. 1272-1286. https://doi.org/10.1158/1055-9965.EPI-11-0035

7. Fidler IJ. The pathogenesis of cancer metastasis: the 'seed and soil' hypothesis revisited. Nature Reviews Cancer. 2003;3(6):453-458. https://doi.org/10.1038/nrc1098

8. Hurst DR, Edmonds MD, Welch DR. Metastamir: the field of metastasis-regulatory microRNA is spreading. Cancer research. 2009;69(19):7495-7498. https://doi.org/10.1158/0008-5472.CAN-09-2111

9. Arakawa K., et al. KEGG-based pathway visualization tool for complex omics data. In silico biology. 2005;5(4):419423.

10. Hulsegge I, Kommadath A, Smits MA. Globaltest and GOEAST: two different approaches for Gene Ontology analysis. In BMC proceedings. 2009. BioMed Central. https://doi.org/10.1186/1753-6561-3-s4-s10

11. Huang DW, Sherman BT, Lempicki RA. Systematic and integrative analysis of large gene lists using DAVID bioinformatics resources. Nature protocols. 2009;4(1):44-57. https://doi.org/10.1038/nprot.2008.211

12. Sheng $\mathrm{W}-\mathrm{Z}$, et al. MicroRNA-21 promotes phosphatase gene and protein kinase B/phosphatidylinositol 3-kinase expression in colorectal cancer. World journal of gastroenterology. 2016;22(24):5532. https://doi.org/10.3748/wjg.v22.i24.5532

13. Longqiu $Y$, et al. A miRNAs panel promotes the proliferation and invasion of colorectal cancer cells by targeting GABBR1. Cancer medicine. 2016;5(8):2022-2031. https://doi.org/10.1002/cam4.760

14. Bakirtzi K, et al. Neurotensin signaling activates microRNAs-21 and-155 and Akt, promotes tumor growth in mice, and is increased in human colon tumors. Gastroenterology. 2011;141(5):1749-1761. https://doi.org/10.1053/j.gastro.2011.07.038

15. Qin J, Luo M. MicroRNA-221 promotes colorectal cancer cell invasion and metastasis by targeting RECK. FEBS letters. 2014;588(1):99-104. https://doi.org/10.1016/j.febslet.2013.11.014

$\diamond \diamond \diamond \diamond \diamond \diamond \diamond$

http://www.ejgm.co.uk 